\title{
Knockdown of ROS1 gene sensitizes breast tumor growth to doxorubicin in a syngeneic mouse model
}

\author{
SNIGDHA TIASH* ${ }^{*}$ MING JANG CHUA ${ }^{*}$ and EZHARUL HOQUE CHOWDHURY \\ Advanced Engineering Platform (AEP) and Jeffrey Cheah School of Medicine and Health Sciences, \\ Monash University (Sunway campus), Petaling Jaya, Selangor 46150, Malaysia
}

Received December 3, 2015; Accepted January 5, 2016

DOI: $10.3892 /$ ijo.2016.3452

\begin{abstract}
Treatment of breast cancer, the second leading cause of female deaths worldwide, with classical drugs is often accompanied by treatment failure and relapse of disease condition. Development of chemoresistance and drug toxicity compels compromising the drug concentration below the threshold level with the consequence of therapeutic inefficacy. Moreover, amplification and over-activation of proto-oncogenes in tumor cells make the treatment more challenging. The oncogene, ROS 1 which is highly expressed in diverse types of cancers including breast carcinoma, functions as a survival protein aiding cancer progression. Thus we speculated that selective silencing of ROS1 gene by carriermediated delivery of siRNA might sensitize the cancer cells to the classical drugs at a relatively low concentration. In this investigation we showed that intracellular delivery of $c$-ROS1targeting siRNA using $\mathrm{pH}$-sensitive inorganic nanoparticles of carbonate apatite sensitizes mouse breast cancer cells (4T1) to doxorubicin, but not to cisplatin or paclitaxel, with the highest enhancement in chemosensitivity obtained at $40 \mathrm{nM}$ of the drug concentration. Although intravenous administrations of ROS1-loaded nanoparticles reduced growth of the tumor, a further substantial effect on growth retardation was noted when the mice were treated with the siRNA- and Dox-bound particles, thus suggesting that silencing of ROS1 gene could sensitize the mouse breast cancer cells both in vitro and
\end{abstract}

Correspondence to: Dr Ezharul Hoque Chowdhury, Jeffrey Cheah School of Medicine and Health Sciences, Monash University, Sunway campus, Jalan Lagoon Selatan, Bandar Sunway, Petaling Jaya, Selangor 46150, Malaysia

E-mail: md.ezharul.hoque@monash.edu

*Contributed equally

Abbreviations: Cis, cisplatin; Dox, doxorubicin; MAPK, mitogen activated protein kinase; NP, nanoparticles; Pax, paclitaxel; PI-3K, phosphatidylinositol-4,5-bisphosphate 3-kinase; siRNA: small interfering RNA

Key words: carbonate apatite nanoparticle, doxorubicin, cisplatin, paclitaxel, ROS1, siRNA, breast cancer, tumor regression in vivo to doxorubicin as a result of synergistic effect of the gene knockdown and the drug action, eventually preventing activation of the survival pathway protein, AKT1. Our findings therefore provide valuable insight into the potential cross-talk between the pathways of ROS1 and doxorubicin for future development of effective therapeutics for breast cancer.

\section{Introduction}

The overpowering growth of cancer cells in a tumor is associated with development of profound complexity in tumor microenvironment with a consequence of developing chemoresistance and metastasis that leads to treatment failure, progressive morbidity and mortality. Breast cancer is the second leading cause of death among women worldwide with chemo-/radiotherapy being the most preferred strategy to combat the disease after surgical removal of the cancerous tissues. A large number of chemical drugs with dissimilar structures and mode of actions are used to treat solid tumors like breast cancer. The updated list of the FDA-approved drugs for treating breast cancer consists of at least 24 drugs with different formulations for combinatorial therapy. Among them, three of the most widely used anticancer drugs: doxorubicin, cisplatin and paclitaxel from three different families were used separately in this study. All of the three drugs enter into cells via passive or carrier-mediated diffusion and act as substrates for the ATP binding cassette multidrug transporters that extrude the drugs out of the cells. The drugs are known to exert many adverse effects principally caused by off-target toxicity of anti-cancer drugs affecting particularly highly dividing healthy cells of the body.

Although the above drugs are currently used in different combinations with other drugs for better treatment outcome, non-specific distribution all over the body causing inadequate drug concentration in the tumor microenvironment diminishes the chemo-effectiveness. Toxicity to healthy tissues compromises the doses and frequencies of treatment resulting in therapeutic failure. Moreover, intrinsic and acquiring multi- drug resistance (MDR) by genetic and epigenetic modifications of cancer cells nullifies the effects of many therapies. In tumor cells, many oncogenes overexpressed or amplified, are involved in cell survival and proliferation abetting cancer progression and minimizing therapeutic activity of the drugs. Thus knocking these genes down using specific siRNA would 
sensitize cancer cells to conventional drugs by reducing the effective threshold concentration of drugs inside the cells.

The proto-oncogene ROSI encoding for a type-I integral membrane protein with tyrosine kinase activity having roles in growth and differentiation, is highly expressed in a variety of tumor cells (1-5). The tumorigenicity assay of human breast cancer cells identified an activated form of ROS1 gene encoding for the extracellular domain as a result of a loss of segment from the gene $(6,7)$, thus making it a potential molecular target to be silenced for breast cancer treatment.

Activation of a receptor tyrosine kinase (RTK) triggers phosphatidylinositol 3-kinase (PI3K) survival pathway stimulating numerous downstream proteins and thereby, regulating key signaling pathways in many cellular processes, such as differentiation, cell survival, transformation, growth, proliferation, apoptosis, motility and cytoskeletal rearrangement. Oncogenic alterations of this pathway were reported in different cancers including breast cancer, offering it as a valuable therapeutic target (8-19). Furthermore, mitogen activated protein kinase (MAPK) pathway also plays a pivotal role in transducing signals in response to extracellular cues, modulating numerous physiological processes, i.e., cell proliferation, growth, differentiation, stress response, immune response, cell migration and apoptosis (20). The activation of MAPK pathway is involved in cancer growth and metastasis in many cancers including breast cancer (21-23). There is fine-tuned cross-talk between the survival and mitogenic pathways, thus endowing cells with capabilities for processing and decoding signals from the multiple receptors activated by different growth factors (24). Stimulation of the cells expressing chimeric receptors that contain the ROS1 kinase domain fused to the ligand binding domain of EGFR, with the corresponding growth factors was eventually found to activate various combinations of PI3K/AKT and MAPK/ERK signaling components, indicating the plausible roles of ROS1 in these pathways (25). Therefore, the level of these pathway proteins could be altered by silencing ROSI expression in cancer cells through delivery of anti-ROS1 siRNA.

However, a suitable nano-carrier to transport siRNA and drugs together inside tumor cells and subsequently release them in cytoplasm is one of the utmost needs for achieving the optimal therapeutic outcomes. The recently developed pH-sensitive inorganic nanoparticles (NP) of carbonate apatite with high binding affinity for nucleic acid therapeutics and conventional drugs have the potential for combined intracellular delivery of the diverse drugs via endocytosis and their fast release at endosomal acidic $\mathrm{pH}$ (26-31). Moreover, this carrier has been used successfully to deliver classical anti-cancer drugs and molecular therapeutics (both transgene and siRNA) in animal models of solid tumors including breast cancer (32-34). Co-delivery of siRNA against ROS1 gene and a classical drug, paclitaxel into human breast cancer cells (MCF-7) showed synergistic cytotoxic effects suggesting that knockdown of ROS1 gene sensitized the cells to the drug (30). Here we reveal that carbonate apatite-mediated delivery of ROS1 siRNA enhanced the chemosensitivity of doxorubicin at $40 \mathrm{nM}$ in murine mammary carcinoma cells (4T1) and significantly reduced $4 \mathrm{~T} 1$ cell-induced tumor volume in a syngeneic mouse model compared to only particles and particle-bound siRNA groups, with a substantial decrease in phosphorylated form of AKT1 protein in PI3K pathway. Nevertheless, silencing of ROS1 gene could not sensitize the 4T1 cells to either cisplatin or paclitaxel.

\section{Materials and methods}

Materials. Dulbecco's modified Eagle's medium (DMEM), calcium chloride dehydrate $\left(\mathrm{CaCl}_{2} \cdot 2 \mathrm{H}_{2} \mathrm{O}\right)$, sodium bicarbonate $\left(\mathrm{NaHCO}_{3}\right)$, thiazolyl blue tetrazolium bromide (MTT), dimethyl sulphoxide (DMSO), Nonidet P-40 (NP-40), dithiothreitol (DTT), protease inhibitor cocktail, phosphatase inhibitor, bovine serum albumin (BSA), sodium chloride $(\mathrm{NaCl})$, Tris base and the anti-cancer drugs doxorubicin hydrochloride (Dox), cis-diammine platinum (II) dichloride (Cis) and paclitaxel (Pax) were purchased from Sigma-Aldrich (St. Louis, MO, USA). Dox and Cis were dissolved in miliQ water ( $2 \mathrm{mM}$ stock) and Pax was dissolved in DMSO (10 mM stock). DMEM powder, fetal bovine serum (FBS), trypsin-ethylenediamine tetraacetate (trypsin-EDTA) and penicillin-streptomycin were obtained from Gibco BRL (Carlsbad, CA, USA). Functionallyvalidated siRNA $(1 \mathrm{nmol})$ against ROS1 gene with target sequence of 5'-AAGGTAATTGCTCTAACTTTA-3' and AllStars Negative Control siRNA in lyophilized forms were procured from Qiagen (Valencia, CA, USA). The siRNAs were reconstituted in RNase-free water to obtain $20 \mu \mathrm{M}$ stock solution. Bradford assay kit, nitrocellulose membrane $(0.45 \mu \mathrm{M})$, Tween-20, Pierce ECL western blot detection reagents were from Bio-Rad Laboratories, Inc. (Hercules, CA, USA). All the four different monoclonal IgG primary antibodies raised in rabbit for phospho-p42/44, total p42/44, phopho-AKT and total AKT, and the horseradish peroxidase conjugated secondary goat anti-rabbit IgG antibody were from Cell Signaling Technology, Inc. (Beverly, MA, USA).

Preparation of carbonate apatite NP and complexing with drugs and siRNA for in vitro study. Synthesis of carbonate apatite NP and their complexing with drugs in presence or absence of ROS1 siRNA were accomplished according to the protocol described previously (30). Briefly, particles were prepared by mixing $4 \mathrm{mM}$ ( $4 \mu \mathrm{l}$ of $1 \mathrm{M}$ stock) of exogenous calcium chloride $\left(\mathrm{CaCl}_{2}\right)$ with $1 \mathrm{ml}$ of the freshly prepared DMEM, pH 7.4, followed by incubation at $37^{\circ} \mathrm{C}$ for $30 \mathrm{~min}$. For complexing of NP with drugs, $4 \mathrm{mM}$ of $\mathrm{CaCl}_{2}$, and 8 or $40 \mathrm{nM}$ of one of the three drugs (Dox, Cis or Pax) were added with or without $10 \mathrm{nM}$ of ROS1 siRNA (NP-drug/NP-siRNAdrug) to $1 \mathrm{ml}$ of DMEM media prior to $30 \mathrm{~min}$ incubation at $37^{\circ} \mathrm{C}$. siRNA-loaded NP (NP-siRNA) was prepared in the same manner, except that no drug was added. Free drugs were maintained in DMEM media without any exogenous $\mathrm{CaCl}_{2}$ and incubated for $30 \mathrm{~min}$. In another set of experiment, NPs were formed in the presence of $1 \mathrm{pM}$ to $10 \mathrm{nM}$ of negative siRNA. Further growth of particles following the incubation period was prevented by adding $10 \%$ FBS. Finally, the cells were treated with the different formulations for a consecutive period of $48 \mathrm{~h}$ prior to cell viability assessment by MTT assay and detection of downstream signaling proteins by western blotting.

Maintenance of mouse breast cancer cell line and cell viability assay by 3-(4,5-dimethylthiazol-2-yl)-2,5-diphenyltetrazolium 
bromide $(M T T)$. Mouse breast cancer cell line, 4T1 was cultured in $75 \mathrm{~cm}^{2}$ tissue culture flasks and maintained in DMEM media (pH 7.4) supplemented with $10 \%$ FBS and $1 \%$ penicillin and streptomycin antibiotic, in a $37^{\circ} \mathrm{C}$ incubator humidified with $5 \% \mathrm{CO}_{2}$. One day before the treatment, cells from exponential growth phase were seeded on a 24-well plate with each of the wells allowed to have approximately $5 \times 10^{4}$ cells. After $24 \mathrm{~h}$, the cells were treated with media only (untreated), NP, free $\operatorname{drug}(\mathrm{s}), \mathrm{NP}-\operatorname{drug}(\mathrm{s}), \mathrm{NP}-\mathrm{siRNA}$ or NP-siRNA-drug(s) for two days. After completing the incubation, $50 \mu \mathrm{l}$ of MTT $(5 \mathrm{mg} / \mathrm{ml}$ in PBS) was added to each well and the plate was left in the incubator for $4 \mathrm{~h}$ for formation of formazan crystals by metabolically active cells. Dimethyl sulfoxide (DMSO) (300 $\mu \mathrm{l})$ was added by replacing the media and plates were agitated on built-in plate shaker for $20 \mathrm{sec}$ to dissolve the dark blue crystals. For control, cells were kept untreated in media containing no exogenous $\mathrm{CaCl}_{2}$. Formazan quantification in the form of optical density (OD) was performed at $595 \mathrm{~nm}$ wavelength with $630 \mathrm{~nm}$ of reference wavelength using a microplate reader. The cell viability for treated cells was calculated using the following equation:

$$
(\%) \text { of cell viability }(\mathrm{CV}) \frac{O D \text { treated }-O D \text { reference }}{O D \text { untreated }-O D \text { reference }} \times 100
$$

Each experiment was done in triplicate and expressed in graphs as mean \pm SD of $\%$ of cell viability.

Formulation of particles for in vivo study. Particles were formed by mixing of $4 \mu \mathrm{l}$ of $1 \mathrm{M} \mathrm{CaCl}_{2}$ in $100 \mu \mathrm{l}$ of freshly prepared bicarbonated ( $44 \mathrm{mM})$ DMEM media and incubating at $37^{\circ} \mathrm{C}$ for $30 \mathrm{~min}$. For complexing with Dox and siRNA (NP-siRNA-Dox), $0.34 \mathrm{mg} / \mathrm{kg}$ of Dox and $50 \mathrm{nM}$ siRNA (20 or $40 \mathrm{nM}$ siRNA was used to prepare complexes for intratumoral injection for downstream protein expression study) were used before incubation. NP-Dox and NP-siRNA were prepared in the same way in the absence of siRNA and Dox, respectively. The control and sample solutions $(100 \mu \mathrm{l})$ were intravenously injected into mice.

Effects of drug-/siRNA-loaded NP on tumor regression in 4T1-induced breast cancer model. Twenty-four female Balb/c mice, aged 6-8 weeks, weighing 15-20 g were purchased and maintained in 12:12 light: dark condition by providing them ad libitum chow and water. All experiments were done in accordance with the regulations imposed by Monash University Animal Welfare Committee. Approximately $1 \times 10^{5} 4 \mathrm{~T} 1$ cells (in $100 \mu \mathrm{l}$ PBS) were injected subcutaneously on the mammary pad of mice (considered as day 1) and the mice were checked regularly for outgrow th of tumor by touching the area of injection. When the volume of the outgrowth reached an average $13.20 \pm 2.51 \mathrm{~mm}^{3}$, mice were grouped in different assemblies (6 mice per group) randomly and treated intravenously (tail-vein) at the right or left caudal vein. The second dose was administered after 3 days from the 1 st dose. The gross body weights of mice were monitored and the lengths and widths of the tumors were measured using the vernier caliper in $\mathrm{mm}$ scale for 30 days while the mice were monitored for their activities.
The volume of the tumor was calculated using the following formula:

$$
\text { Tumor volume }\left(\mathrm{mm}^{3}\right)=1 / 2\left(\text { Length } \mathrm{x} \mathrm{Width}^{2}\right)
$$

The data are presented here as the mean \pm SD of tumor volume from each group.

Separate sets of mice were treated with $100 \mu \mathrm{l}$ of free/ NP-Dox $(0.34 \mathrm{mg} / \mathrm{kg})$, NP-siRNA (20 or $40 \mathrm{nM}$ ) and NP-DoxsiRNA intratumorally when the tumor volume reached $75 \mathrm{~mm}^{3}$, with two doses per week over a period of 14 days. After treatment, the mice were sacrificed and tumors were excised and processed for western blot analysis.

Western blot analysis for PI3K and MAPK pathways in vitro and in vivo. Cells treated with NP,Dox, NP-Dox, NP-siRNA and NP-Dox-siRNA for two consecutive days were washed in prechilled PBS and lysed in lysis buffer (1\% NP40, $9.3 \mathrm{mM}$ DTT in PBS, 1:1000 protease inhibitor and 1:100 phosphatase inhibitor) pipetting up and down. The cell lysates were centrifuged at $13,000 \mathrm{rpm}$ for $10 \mathrm{~min}$ at $4^{\circ} \mathrm{C}$ to remove cellular debris. The protein extracts were collected from supernatant and subjected to Quick Start Bradford Protein Assay (according to instructions provided by the manufacturer; Bio-Rad Laboratories, Inc.) for estimating the total proteins. Moreover, the excised tumor tissues from treated mice were lysed in lysis buffer using tissue homogenizer (Omni International, Kennesaw, GA, USA) and centrifuged for $15 \mathrm{~min}$ at $15,000 \mathrm{rpm}$ at $4^{\circ} \mathrm{C}$ to sediment the cellular and tissue debris. Tumor tissue lysate from each group of mice were pooled and protein content was measured by Bradford assay. All the samples were aliquoted and stored at $-80^{\circ} \mathrm{C}$ for further study.

The protein extracts were subjected to SDS-PAGE $(7.5 \%$ gel) at $200 \mathrm{~V}$ for $40 \mathrm{~min}$ and the resolved proteins were electro-transferred for $12 \mathrm{~min}$ at $18 \mathrm{~V}$ to nitrocellulose membranes (Thermo Fisher Scientific, Waltham, MA, USA) using western blotting semi-dry transfer unit (Hoefer, San Francisco, CA, USA). The membranes were blocked for $1 \mathrm{~h}$ at room temperature with TBST (10X buffer; $200 \mathrm{mM}$ Tris base, $1.5 \mathrm{M} \mathrm{NaCl}$ in MiliQ water; $0.1 \%$ Tween-20 was added and $\mathrm{pH}$ was adjusted to 7.5 for $1 \mathrm{X}$ buffer)-containing $5 \%$ bovine serum albumin (BSA). The membranes were then incubated with one of the four primary antibodies in TBST with $5 \%$ BSA overnight at $4^{\circ} \mathrm{C}$ with gentle shaking. Membranes were washed twice with TBST for $10 \mathrm{~min}$ each and further incubated with secondary antibody (1:5000) for $1 \mathrm{~h}$ at room temperature. Then the membrane was washed two times for $10 \mathrm{~min}$ each with TBST to remove any nonbound secondary antibody. The membrane was incubated with Pierce ECL Western Blotting Detection Reagent (Bio-Rad Laboratories, Inc.) at room temperature for $5 \mathrm{~min}$. Excess detection reagent was drained off and the membrane was exposed to X-ray film placed on top of the membrane for an appropriate time in an X-ray film cassette (Amersham, Piscataway, NJ, USA). Membranes were also tested for equal loading.

Statistical analysis. Statistical analysis was done using the SPSS (version 17 for Windows) for in vivo (tumor regression study) data. LSD post-hoc test for one way ANOVA was used 

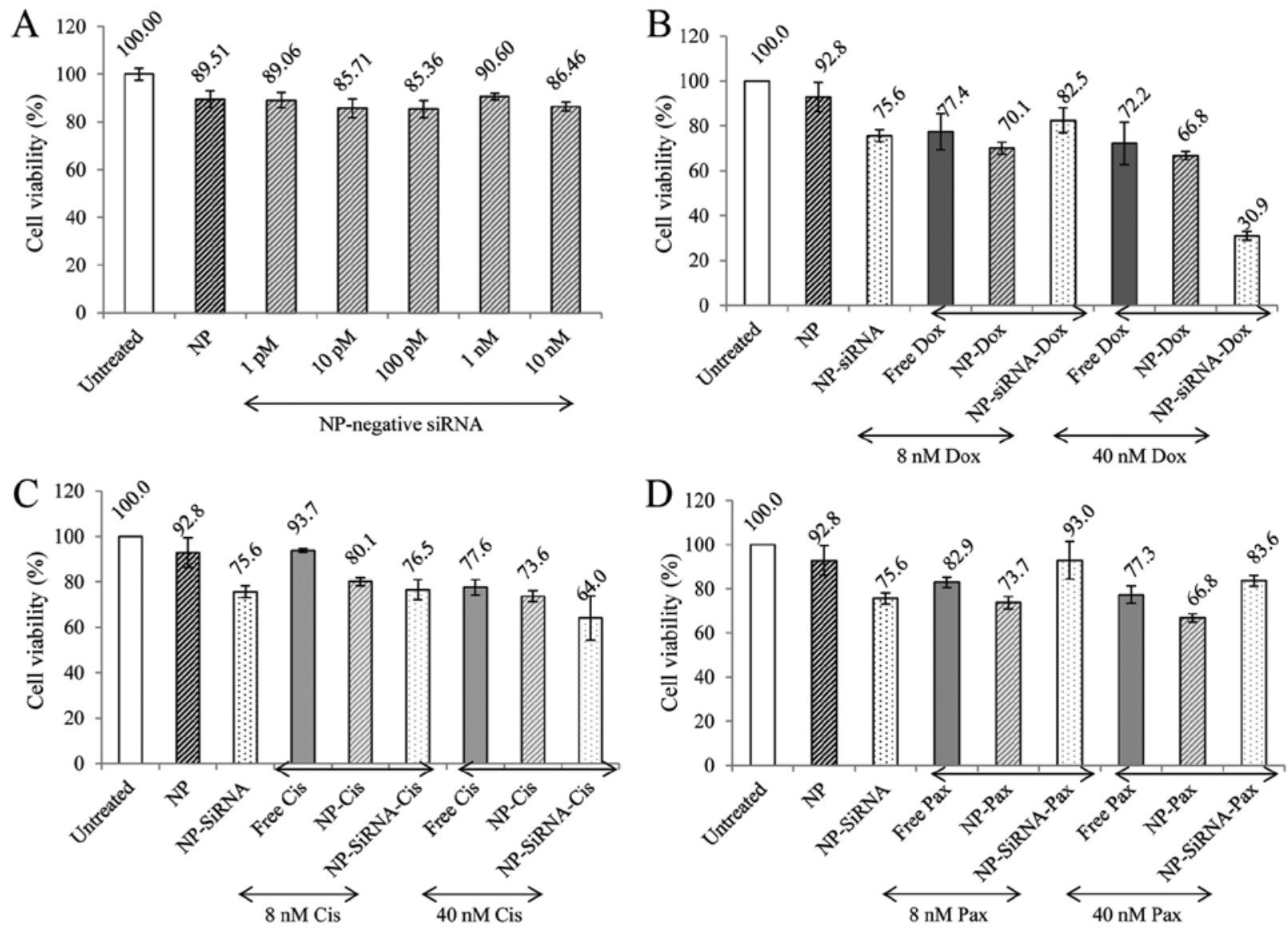

Figure 1. Effect of NP-bound siRNA and different drug treatments on 4T1 cell viability. Cells treated with (A) NP-bound negative control siRNA from 1 pM to $10 \mathrm{nM}$; (B) NP-bound Dox, ROS1 siRNA, Dox+ ROS1 siRNA (C) NP-bound Cis, ROS1 siRNA, Cis + ROS1 siRNA and (D) NP-bound Pax, ROS1 siRNA, $\mathrm{Pax}+$ ROS1 siRNA. Values are presented as the mean $\pm \mathrm{SD}$ of $\%$ of cell viability compared to untreated cells for triplicate samples.

to analyze and compare the significant difference between different treatment groups. Data were considered statistically significant at $\mathrm{p}<0.05$.

\section{Results}

Influences of particle-bound drugs and ROS1 siRNA on cell viability. Fig. 1A depicts the viability of mouse breast cancer (4T1) cells treated with different concentrations of 'Allstars Negative Control siRNA' ( $1 \mathrm{pM}$ to $10 \mathrm{nM}$ ) originally used to complex with carbonate apatite NP with apparently no alteration in cell viability when incubated for two consecutive days. The influences of the NP loaded with siRNA against $c$-ROSI gene and Dox (NP-siRNA-Dox) on cell viability is shown in Fig. 1B. Treatment of $4 \mathrm{~T} 1$ cells with the NP formed with $4 \mathrm{mM}$ of exogenous $\mathrm{CaCl}_{2}$ and fixed concentrations of endogenous inorganic phosphate $(0.9 \mathrm{mM})$ and bicarbonate $(44 \mathrm{mM})$ showed $92.84 \pm 6.61 \%$ of viability compared to the untreated cells and therefore the same conditions were maintained for forming complexes with drugs and siRNA in the subsequent in vitro studies. The NP-siRNA treatment rendered the cells less viable reducing the viability to $75.6 \pm 2.61 \%$ compared to the untreated cells whereas the NP-Dox formulation was found slightly less viable than free Dox at both 8 and $40 \mathrm{nM}$ of drug concentrations. Interestingly, the NP-siRNA-Dox complex formed at $40 \mathrm{nM}$ of Dox markedly reduced the cell viability to $30.93 \pm 1.88 \%$ compared to the untreated cells. On the contrary,

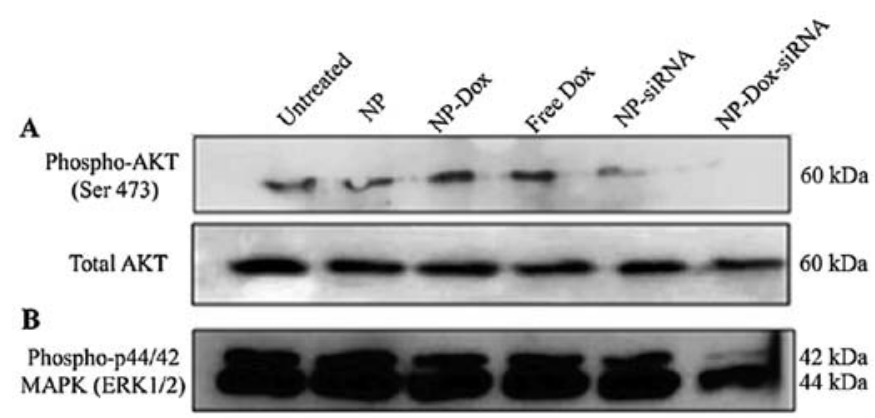

Figure 2. Effects of co-delivery of $c$-ROS1 siRNA and Dox on AKT and MAPK activation in $4 \mathrm{~T} 1$ cells. After two days of consecutive treatment, cell lysates were subjected to western blot for detection of (A) phospho- and total AKT or (B) phosphor-p44/42 MAPK (ERK1/2).

the NP-siRNA-Dox complex formed at $8 \mathrm{nM}$ of the drug did not result in a decrease in cell viability (Fig. 1B).

As shown in Fig. 1C, NP-Cis treatment abated cell viability compared to free Cis predominantly at lower concentration of Cis $(8 \mathrm{nM})$. However, the NP-siRNA-Cis treatment was not accompanied by significant cytotoxicity regardless of the concentrations of the drug used. Moreover, although NP-Pax showed more cytotoxicity than free Pax at both low $(8 \mathrm{nM})$ and high concentration $(40 \mathrm{nM})$ of the drug, treatment of the cells with NP-siRNA-Pax did not demonstrate any significant effect (Fig. 1D). 


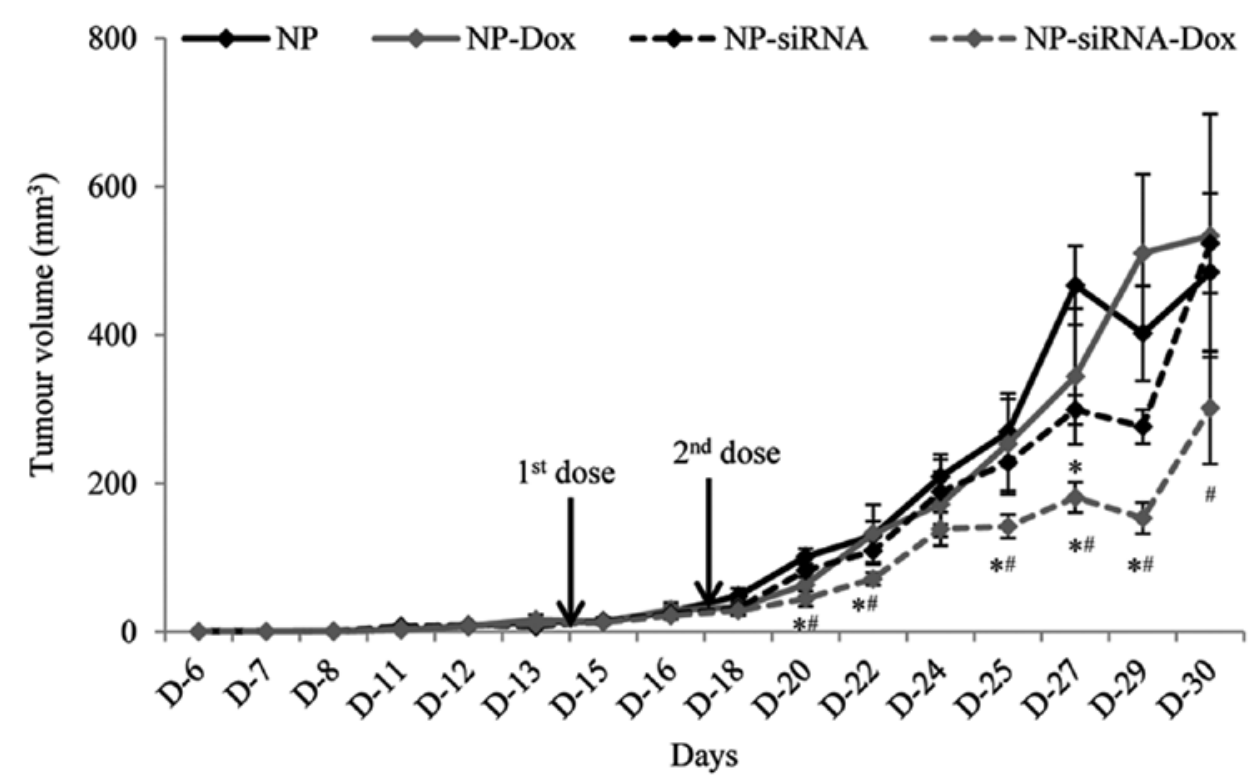

Figure 3. Effects of co-delivery of $c$-ROS1 siRNA and Dox on regression of the 4T1-induced breast tumor. Six mice per group were treated intravenously with NP, NP-Dox $(0.34 \mathrm{mg} / \mathrm{kg}), \mathrm{NP}$-siRNA $(50 \mathrm{nM})$ and NP-siRNA-Dox and data are presented as the mean $\pm \mathrm{SD}$ of tumor volume. Values are significant when " $\mathrm{p}<0.05$ compared to NP group and ${ }^{\#} \mathrm{p}<0.05$ compared to NP-siRNA treated group.

Effects of co-treatment of c-ROS1 siRNA and Dox on AKT and MAPK activation. Since the NP complexes of ROS1 siRNA and Dox $(40 \mathrm{nM})$ exhibited a synergistic effect on cytotoxicity in $4 \mathrm{~T} 1$ cells (Fig. 1B), western blotting was performed to assess the activation status of MAPK or PI-3 kinase pathway by observing the intensity of the phosphorylated form of MAPK or AKT, respectively, following incubation of the cells with the NP-siRNA-Dox complexes for two consecutive days (Fig. 2). As shown in Fig. 2A, treatment of the cells with siRNA-loaded NP (NP-siRNA) reduced the intensity of phosphorylated AKT (Phospho-AKT) (Ser473) compared to the untreated or NP-treated cells, although free Dox and NP-Dox did not change it. Surprisingly, the combined delivery of siRNA and Dox (NP-siRNA-Dox) caused the disappearance of phosphoAKT in the treated cells, while the total AKT level remained unchanged. The combined treatment also decreased the level of phosphorylated ERK1 (42 kDa) protein in MAPK pathway, whereas the effect of free Dox, NP-Dox or NP-siRNA was not so significant (Fig. 2B).

Effects of NP-facilitate combined delivery of c-ROSI siRNA and Dox on tumor regression. As delivery of siRNA against $c$-ROS1 gene induces the highest chemosensitivity of 4T1 cells to Dox (Fig. 1B), NP-siRNA-Dox complexes were intravenously administered in mice bearing 4T1-induced breast tumors to confirm antitumor activity of the drug in vivo. Fig. 3 showed the influences of the NP loaded with Dox, siRNA or both on tumor progression over a period of time. The average body weight of mice showed no observable variance with time among the different groups during tumor development and treatment period (data not shown). The tumor was palpable at day 6 to 9 after subcutaneous inoculation of the tumor cells. NP-Dox treated mice showed similar tumor outgrowth with time as the NP-treated mice, whereas NP-siRNA treatment showed relatively slow development of tumors in mice compared to the NP alone, although the

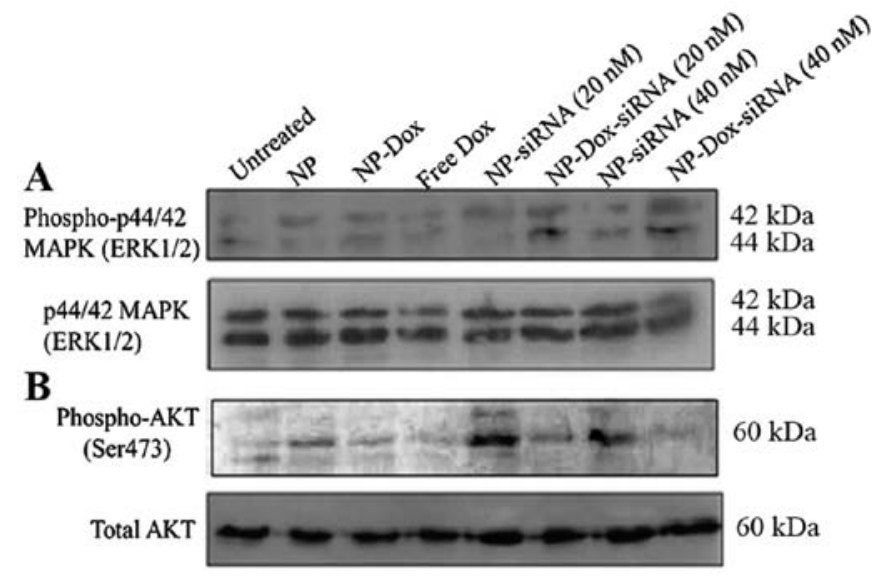

Figure 4. Effects of co-delivery of c-ROS1 siRNA and Dox on AKT and MAPK activation in tumor tissues. After 14 days of intratumor treatment, tumor tissue lysates were subjected to western blotting for detection of (A) phospho- and total p44/42 MAPK (ERK1/2) or (B) phospho- and total AKT.

difference was not significant except for day $27(\mathrm{p}=0.011)$. When the mice were treated with Dox- and siRNA-embedded particles (NP-siRNA-Dox), the tumor volumes were observed to drastically decline particularly after the 2 nd treatment on day 17 compared to other groups.

Effects of co-delivery of ROS1 siRNA and Dox on AKT and $M A P K$ activation in the tumor. In an attempt to correlate the chemosensitizing effect of ROSI gene knockdown on substantial tumor regression, to the activation of PI-3 kinase or MAP kinase pathway, western blot analysis was carried out in the tumor lysates, following intratumoral delivery of free Dox, NP, NP-Dox, NP-siRNA and NP-Dox-siRNA. However, unlike the in vitro scenario, the combined delivery of siRNA and Dox in vivo did not successfully block the phosphorylation of ERK 


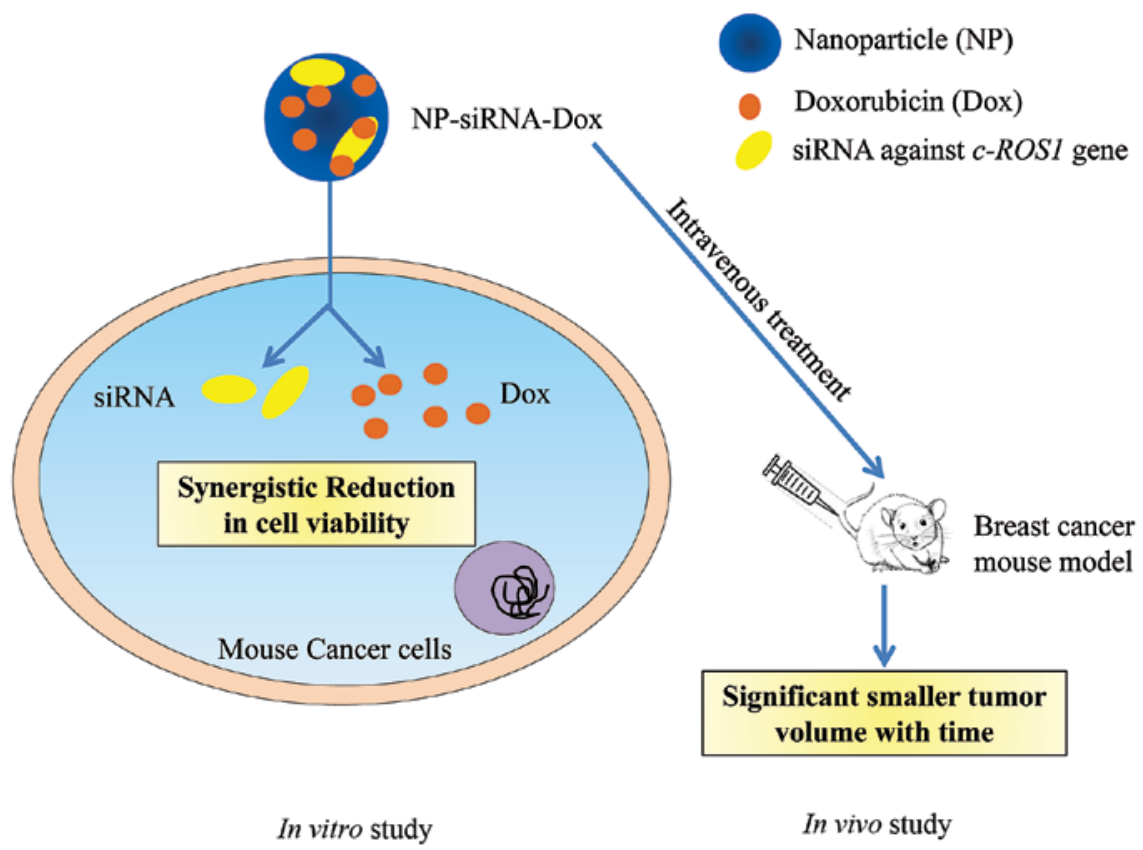

Figure 5. Summary illustration of the findings. Knockdown of ROS1 gene sensitizes cancer cells to Dox and thus show higher cytotoxicity (in vitro) and slower growth of tumors (in vivo) when treated with both ROS1-targeting siRNA and Dox loaded NP.

protein in MAPK pathway (Fig. 4A). On the contrary, the same treatment was found to have an effect on reducing the phosphorylated AKT1 (Ser473) level particularly at higher siRNA concentration (40 nM) compared to NP-siRNA (Fig. 4B), which is consistent with the effect observed in vitro.

\section{Discussion}

Naked siRNA being anionic in nature is electrostatically repelled by anionic cell membrane and also subjected to degradation by endonucleases present in intracellular acidic vesicles as well as the blood stream, therefore necessitating an efficient carrier to carry and release them to the target site (the cytosol). The $\mathrm{pH}$ sensitive inorganic particles of carbonate apatite have been established as smart delivery vehicles to transport siRNAs both in vitro and in animal models for silencing a diverse range of genes (28-30,33-36). Assessment of cell viability using particle-loaded 'AllStars Negative Control siRNA', a scrambled siRNA that has no target mRNA sequence, demonstrated no apparent cytotoxic effect irrespective of the siRNA concentrations used (Fig. 1A), signifying the potential role of siRNA in gene-specific knockdown and subsequent alteration in cell functionality. The reduction in cell viability upon intracellular delivery of the functionallyvalidated siRNA targeting ROS1 gene (Fig. 1B) could be due to the knockdown of ROS1 mRNA transcript, thus preventing the production of ROS1 receptor tyrosine kinase and the activation of its possible downstream signaling cascades, such as MAPK or PI-3 kinase pathway. However, NP-assisted delivery of ROS1 siRNA in 4T1 cells resulted in dephosphorylation of AKT, but not MAPK (Fig. 2), suggesting that PI-3 kinase pathway might be a predominant downstream signaling cascade of ROS1 receptor tyrosine kinase in this particular cell line. The absence of dephosphorylated form of AKT1 in tumor lysate upon treatment with NP-siRNA (Fig. 4) could be due to the inefficient knockdown of ROS1 gene owing to the existence of barriers to siRNA delivery in vivo. Similar effect of declining cell viability was observed in MCF-7 cells pretreated with NP-loaded ROS1 siRNA for 48 h (30). Moreover, ROS1 inhibitor was also found to play an antiproliferative role in lung cancer cells and antitumor activity in lung cancer patients $(37,38)$.

Carbonate apatite particles have been shown in earlier studies to have high binding affinity for structurally different classical drugs and exert more cytotoxic effect and antitumor activity by delivering more drugs in cancer cells through particle-mediated endocytosis in contrast to the free drugs that mainly penetrate the cell membrane via passive diffusion $(32,31)$. However, in this study, NP-Dox treatment did not demonstrate any significant decrease in cell viability compared to free Dox, probably because of the inadequate amount of the drug ( 8 and $40 \mathrm{nM}$ ) used, which was not sufficient enough to effectively kill these aggressive mouse breast cancer cells. However, knockdown of ROS1 gene was found to sensitize the cells to Dox, as evident from the enhanced cytotoxic effect following co-delivery of ROS1 siRNA and Dox with the help of NP (NP-siRNA-Dox). This could be correlated to the substantial decrease in phosphorylated AKT and ERK proteins (Fig. 2) of PI-3 kinase and MAPK pathways, respectively, by the treatment with NP-siRNA-Dox. Despite the apparent inefficacy of ROS1 siRNA to deactivate phosphorylated MAPK, the co-delivery of Dox and ROS1 siRNA suppressed the MAPK activation (Fig. 2), suggesting that Dox and ROS1 gene knockdown might synergize to dephosphorylate MAPK. It was found earlier that inhibition of PI3K/AKT repressed resistance of cells to Dox (39) and activation of MAPK pathway conferred resistance to Dox, as these pathway proteins induce the expression of drug transporter proteins responsible for extruding drugs from inside to outside of the cell and thus, developing drug resistance (40-42). Targeted cleavage of ROS1 
mRNA might not only assist in declining cell proliferation/ survival, but also reduce Dox resistance through inactivation of PI-3 kinase and MAPK pathways, thereby rendering the cells more responsive to Dox. Similar findings were observed in vivo with a noticeable and persistent decline in tumor growth following intravenous injection of NP-siRNA-Dox (Fig. 3). However, only the band for phospho-AKT, but not phosphoMAPK disappeared in the tumor treated with NP-siRNA-Dox, which could be explained by the inefficient delivery of the siRNA as well as the drug to the tumor, or the difference in activation level of the two pathways depending on the in vitro and in vivo conditions.

In summary, simultaneous delivery of ROS1 siRNA and Dox using carbonate apatite NPs into 4T1 breast cancer cells and the established tumor in a syngeneic mouse model sensitized the cancer cells, and tumor to Dox (Fig. 5) by silencing the ROS1 gene expression and predominantly downregulating PI-3 kinase pathway, thereby enabling the killing of cancer cells at very low concentrations of the drug. Toxicological and immunological studies in future are needed to confirm the applicability of this combination in breast cancer treatment. This novel approach has extraordinary clinical prospects in tumor therapy to efficiently and selectively destroy cancer cells and circumvent the drug-induced toxicity in normal healthy cells.

\section{Acknowledgements}

The study was supported by a research grant (FRGS/2/2013/ SG05/MUSM/02/2) of the Ministry of Higher Education (MOHE), Malaysia.

\section{References}

1. Trent JM, Rosenfeld SB and Meyskens FL: Chromosome 6q involvement in human malignant melanoma. Cancer Genet Cytogenet 9: 177-180, 1983.

2. Atkin NB and Baker MC: Specific chromosome change in ovarian cancer. Cancer Genet Cytogenet 3: 275-276, 1981.

3. Watkins D, Dion F, Poisson M, Delattre JY and Rouleau GA: Analysis of oncogene expression in primary human gliomas: Evidence for increased expression of the ros oncogene. Cancer Genet Cytogenet 72: 130-136, 1994.

4. Jun HJ, Woolfenden S, Coven S, Lane K, Bronson R, Housman D and Charest A: Epigenetic regulation of c-ROS receptor tyrosine kinase expression in malignant gliomas. Cancer Res 69 2180-2184, 2009.

5. Eom M, Han A, Yi SY, Shin JJ, Cui Y and Park KH: RHEB expression in fibroadenomas of the breast. Pathol Int 58: 226-232, 2008.

6. Fasano O, Birnbaum D, Edlund L, Fogh J and Wigler M: New human transforming genes detected by a tumorigenicity assay. Mol Cell Biol 4: 1695-1705, 1984.

7. Birchmeier C, Birnbaum D, Waitches G, Fasano O and Wigler M: Characterization of an activated human ros gene. Mol Cell Biol 6: 3109-3116, 1986.

8. Neri LM, Borgatti P, Capitani S and Martelli AM: The nuclear phosphoinositide 3-kinase/AKT pathway: A new second messenger system. Biochim Biophys Acta 1584: 73-80, 2002.

9. Vivanco I and Sawyers CL: The phosphatidylinositol 3-Kinase AKT pathway in human cancer. Nat Rev Cancer 2: 489-501, 2002.

10. López-Knowles E, O'Toole SA, McNeil CM, Millar EK, Qiu MR, Crea P, Daly RJ, Musgrove EA and Sutherland RL: PI3K pathway activation in breast cancer is associated with the basal-like phenotype and cancer-specific mortality. Int J Cancer 126: 1121-1131, 2010.

11. Osaki M, Oshimura M and Ito H: PI3K-Akt pathway: Its functions and alterations in human cancer. Apoptosis 9: 667-676, 2004.
12. Bader AG, Kang S, Zhao L and Vogt PK: Oncogenic PI3K deregulates transcription and translation. Nat Rev Cancer 5: 921-929, 2005.

13. Liu W, Bagaitkar J and Watabe K: Roles of AKT signal in breast cancer. Front Biosci 12: 4011-4019, 2007.

14. Engelman JA, Luo J and Cantley LC: The evolution of phosphatidylinositol 3-kinases as regulators of growth and metabolism. Nat Rev Genet 7: 606-619, 2006.

15. Courtney KD, Corcoran RB and Engelman JA: The PI3K pathway as drug target in human cancer. J Clin Oncol 28: 1075-1083, 2010.

16. Castellano E and Downward J: RAS Interaction with PI3K: More than just another effector pathway. Genes Cancer 2: 261-274, 2011.

17. Osaki M, Kase S, Adachi K, Takeda A, Hashimoto K and Ito H: Inhibition of the PI3K-Akt signaling pathway enhances the sensitivity of Fas-mediated apoptosis in human gastric carcinoma cell line, MKN-45. J Cancer Res Clin Oncol 130: 8-14, 2004.

18. Lee CM, Fuhrman CB, Planelles V, Peltier MR, Gaffney DK, Soisson AP, Dodson MK, Tolley HD, Green CL and Zempolich KA: Phosphatidylinositol 3-kinase inhibition by LY294002 radiosensitizes human cervical cancer cell lines. Clin Cancer Res 12: 250-256, 2006

19. Martin-Fernandez C, Bales J, Hodgkinson C, Welman A, Welham MJ, Dive C and Morrow CJ: Blocking phosphoinositide 3-kinase activity in colorectal cancer cells reduces proliferation but does not increase apoptosis alone or in combination with cytotoxic drugs. Mol Cancer Res 7: 955-965, 2009.

20. Roux PP and Blenis J: ERK and p38 MAPK-activated protein kinases: A family of protein kinases with diverse biological functions. Microbiol Mol Biol Rev 68: 320-344, 2004.

21. Santen RJ, Song RX, McPherson R, Kumar R, Adam L, Jeng MH and Yue W: The role of mitogen-activated protein (MAP) kinase in breast cancer. J Steroid Biochem Mol Biol 80: 239-256, 2002.

22. Wu GS: Role of mitogen-activated protein kinase phosphatases (MKPs) in cancer. Cancer Metastasis Rev 26: 579-585, 2007.

23. Keyse SM: Dual-specificity MAP kinase phosphatases (MKPs) and cancer. Cancer Metastasis Rev 27: 253-261, 2008.

24. Aksamitiene E, Kiyatkin A and Kholodenko BN: Cross-talk between mitogenic Ras/MAPK and survival PI3K/Akt pathways: A fine balance. Biochem Soc Trans 40: 139-146, 2012.

25. Davies KD and Doebele RC: Molecular pathways: ROS1 fusion proteins in cancer. Clin Cancer Res 19: 4040-4045, 2013.

26. Chowdhury EH and Akaike T: Bio-functional inorganic materials: An attractive branch of gene-based nano-medicine delivery for 21st century. Curr Gene Ther 5: 669-676, 2005.

27. Chowdhury EH: pH-sensitive nano-crystals of carbonate apatite for smart and cell-specific transgene delivery. Expert Opin Drug Deliv 4: 193-196, 2007.

28. Hossain S, Stanislaus A, Chua MJ, Tada S, Tagawa Y, Chowdhury EH and Akaike T: Carbonate apatite-facilitated intracellularly delivered siRNA for efficient knockdown of functional genes. J Control Release 147: 101-108, 2010.

29. Stanislaus A, Kunnath AP, Tiash S, Fatemian T, Kamaruzman NI, Bakhtiar A, Mustak S, Hossain S, Akaike T and Chowdhury EH: Intracellular delivery of NF- $\kappa B$ small interfering RNA for modulating therapeutic activities of classical anticancer drugs in human cervical cancer cells. Drugs Ther Stud 3 (e7): 28-32, 2013. doi: $10.4081 /$ dts.2013.e7.

30. Chua MJ, Tiash S, Fatemian T, Noordin MI, Keng CS and Chowdhury EH: Carbonate apatite-facilitated intracellular delivery of c-ROS1 small interfering RNA sensitises MCF-7 breast cancer cells to cisplatin and paclitaxel. OA Cancer 1: 1-9, 2013. http://www.oapublishinglondon.com/article/603.

31. Tiash S, Othman I, Rosli R and Chowdhury EH: Methotrexateand cyclophosphamide-embedded pure and strontiumsubstituted carbonate apatite nanoparticles for augmentation of chemotherapeutic activities in breast cancer cells. Curr Drug Deliv 11: 214-222, 2014.

32. Hossain S, Yamamoto H, Chowdhury EH, Wu X, Hirose H, Haque A, Doki Y, Mori M and Akaike T: Fabrication and intracellular delivery of doxorubicin/carbonate apatite nanocomposites: Effect on growth retardation of established colon tumor. PLoS One 8: e60428, 2013.

33. Kunnath AP, Tiash S, Fatemian T, Morshed M, Mohamed SM and Chowdhury EH: Intracellular delivery of ERBB2 siRNA and p53 gene synergistically inhibits the growth of established tumour in an immunocompetent mouse. J Cancer Sci Ther 6: 99-104, 2014. doi: 10.4172/1948-5956.1000256. 
34. Kunnath AP, Kamaruzman NI and Chowdhury EH: Nanoparticle-facilitated intratumoral delivery of Bcl-2/IGF-1R siRNAs and p53 gene synergistically inhibits tumor growth in immunocompetent mice. J Nanomed Nanotechnol S10, 2014. doi: 10.4172/2157-7439.S10-001.

35. Li YT, Chua MJ, Kunnath AP and Chowdhury EH: Reversing multidrug resistance in breast cancer cells by silencing ABC transporter genes with nanoparticle-facilitated delivery of target siRNAs. Int J Nanomed 7: 2473-2481, 2012.

36. Stanislaus A, Bakhtiar A, Salleh D, Tiash S, Fatemian T, Hossain S, Akaike T and Chowdhury EH: Knockdown of PLC-gamma-2 and calmodulin 1 genes sensitizes human cervical adenocarcinoma cells to doxorubicin and paclitaxel. Cancer Cell Int 12: 30, 2012.

37. Davies KD, Le AT, Theodoro MF, Skokan MC, Aisner DL, Berge EM, Terracciano LM, Cappuzzo F, Incarbone M, Roncalli M, et al: Identifying and targeting ROS1 gene fusions in non-small cell lung cancer. Clin Cancer Res 18: 4570-4579, 2012.

38. Shaw AT, Ou SH, Bang YJ, Camidge DR, Solomon BJ, Salgia R, Riely GJ, Varella-Garcia M, Shapiro GI, Costa DB, et al: Crizotinib in ROS1-rearranged non-small-cell lung cancer. N Engl J Med 371: 1963-1971, 2014.
39. Jung KA, Choi BH and Kwak MK: The c-MET/PI3K signaling is associated with cancer resistance to doxorubicin and photodynamic therapy by elevating BCRP/ABCG2 expression. Mol Pharmacol 87: 465-476, 2015.

40. McCubrey JA, Steelman LS, Abrams SL, Lee JT, Chang F, Bertrand FE, Navolanic PM, Terrian DM, Franklin RA, D'Assoro AB, et al: Roles of the RAF/MEK/ERK and PI3K/ PTEN/AKT pathways in malignant transformation and drug resistance. Adv Enzyme Regul 46: 249-279, 2006.

41. McCubrey JA, Steelman LS, Chappell WH, Abrams SL, Wong EW, Chang F, Lehmann B, Terrian DM, Milella M, Tafuri A, et al: Roles of the Raf/MEK/ERK pathway in cell growth, malignant transformation and drug resistance. Biochim Biophys Acta 1773: 1263-1284, 2007.

42. Katayama K, Yoshioka S, Tsukahara S, Mitsuhashi J and Sugimoto Y: Inhibition of the mitogen-activated protein kinase pathway results in the down-regulation of P-glycoprotein. Mol Cancer Ther 6: 2092-2102, 2007. 